\title{
Role of Transesophageal Echocardiography in Perioperative Patient Management of Lung Transplantation Surgery
}

Kathirvel Subramaniam, Stephen A Esper

\begin{abstract}
Lung transplantation is the only option for patients with end-stage lung disease. Chronic obstructive lung disease, idiopathic pulmonary fibrosis, cystic fibrosis and primary pulmonary hypertension are few common indications for lung transplantation. Patients with end-stage lung disease may have pre-existing cardiovascular compromise related to pulmonary hypertension and other cardiovascular lesions, such as coronary artery disease or valvular heart disease. Preoperative evaluation and optimization of hemodynamics is expected to improve outcomes from lung transplantation. Intraoperative hemodynamic instability is common during lung transplantation and requires highest level of cardiovascular monitoring. After transplantation, vascular anastomosis should be evaluated for flow patterns to rule out obstruction from stenosis or thrombosis. Postoperative complications are common and include bleeding, cardiac failure and hypoxemia from right to left shunt. Primary graft dysfunction may necessitate mechanical cardiorespiratory support. Transesophageal echocardiography plays a central role in preoperative evaluation, intraoperative hemodynamic management, evaluation of pulmonary vascular anastomosis, diagnosis of postoperative complications and also in the critical care management of mechanical cardiorespiratory support.
\end{abstract}

Keywords: Lung transplantation, Transesophageal echocardiography, Perioperative management.

How to cite this article: Subramaniam K, Esper SA. Role of Transesophageal Echocardiography in Perioperative Patient Management of Lung Transplantation Surgery. J Perioper Echocardiogr 2013;1(2):48-56.

Source of support: Nil

Conflict of interest: None declared

\section{INTRODUCTION}

Lung transplantation is performed for patients with end stage pulmonary disease. These patients are at risk for cardiopulmonary dysfunction and hemodynamic instability in the perioperative period. Transesophageal echocardiography (TEE) is extremely helpful for perioperative hemodynamic management of patients undergoing lung transplantation. American Society of Anesthesiologists/Society of Cardiovascular Anesthesiologists consensus statement (2010) recommends that TEE may be used when the nature of the planned surgery or the patient's known or suspected cardiovascular pathology might result in severe hemodynamic, pulmonary, or neurologic compromise. ${ }^{1}$ At author's institution, which performs over 100 lung transplants per year, TEE is used in all patients undergoing lung transplantation unless there is a contraindication. To date, there are no outcome studies in the literature to prove the utility of TEE in lung transplantation surgery. Puentes et al studied the impact of TEE on surgical decisionmaking during lung transplantation surgery. New surgical intervention was triggered in $2.6 \%$ of patients by TEE which showed baseline cardiac abnormalities in $50 \%$ of their patients. ${ }^{2}$ In this review, we explain the usefulness of TEE in the perioperative management of lung transplantation recipients.

\section{PREOPERATIVE TEE}

Patients with end-stage lung disease (ESLD) develop pulmonary arterial hypertension (PAH) secondary to their lung pathology. Primary pulmonary vascular hypertension is also an indication for lung transplantation (LTX) surgery. All patients presenting to the anesthesiologist on the day of surgery would have been thoroughly evaluated for PAH in the preoperative period. Evaluation includes right heart catheterization, coronary angiography and echocardiography. Most patients have pretransplant transthoracic echocardiography (TTE) examination but patients with severe PAH or structural heart disease are also evaluated by TEE. TEE provides high resolution images compared to TTE and is especially useful in patients with poor TTE images. Anesthesiologist should review preoperative baseline biventricular function, estimated right ventricular systolic pressure, severity of tricuspid regurgitation, any intracardiac shunt and other valvular/anatomic abnormalities from available echocardiographic studies. Interval changes in ventricular function and pulmonary artery pressure between the time when echocardiography was performed and transplant surgery could be significant in progressive lung disease.

Gorcsan et al evaluated patients with severe PAH waiting for LTX by preoperative TEE and identified additional data which altered surgical therapy in $25 \%$ of their patients. ${ }^{3}$ Two patients underwent pulmonary thromboendarterectomy instead of LTX. Plans were altered to perform combined heart-lung transplantation instead of only double LTX in three other patients. Such major modifications of surgical plans also explain the importance of intraoperative prebypass TEE examination in recipients who had only TTE preoperatively. 


\section{RIGHT VENTRICLE}

Right ventricular (RV) function is the focus of TEE examination during the intraoperative period. Preoperative RV dilatation and dysfunction as well as RV hypertrophy with diastolic dysfunction are common (Fig. 1). Physiologically, stressful intraoperative events like induction of anesthesia, tracheal intubation, positive pressure ventilation, pneumatic tamponade, Trendelenburg position for line placement, hypoxemia, hypercarbia, acidosis and clamping of pulmonary artery (PA) can all cause severe RV dysfunction, leading to cardiogenic shock. Continuous monitoring of RV function can prevent the development of adverse cardiac events during LTX by allowing the operator to diagnose the above discrepancies, guiding therapeutic maneuvers. It is our practice to insert the TEE probe immediately after intubation to evaluate the patient's hemodynamic profile. Right ventricular (RV) failure from pulmonary hypertensive crisis is the most common reason for hemodynamic instability during induction of anesthesia and TEE provides definitive diagnoses in such situations. Initiation of pulmonary vasodilators and inotropes should commence immediately and therapy can be guided by TEE to achieve hemodynamic stability.

Response of RV to clamping of the PA is used to make the surgical decision whether to perform the LTX on cardiopulmonary bypass (CPB) support or by the sequential off CPB method. RV dilatation and dysfunction in response to PA clamp is the indication for CPB. Sometimes, milrinone/ nitric oxide and epinephrine are given to improve RV function before making final decision to proceed with CPB. Again, RV response to this pharmacological treatment is monitored by TEE and no significant improvement with deteriorating hemodynamics will direct the surgeon to initiate $\mathrm{CPB}$.

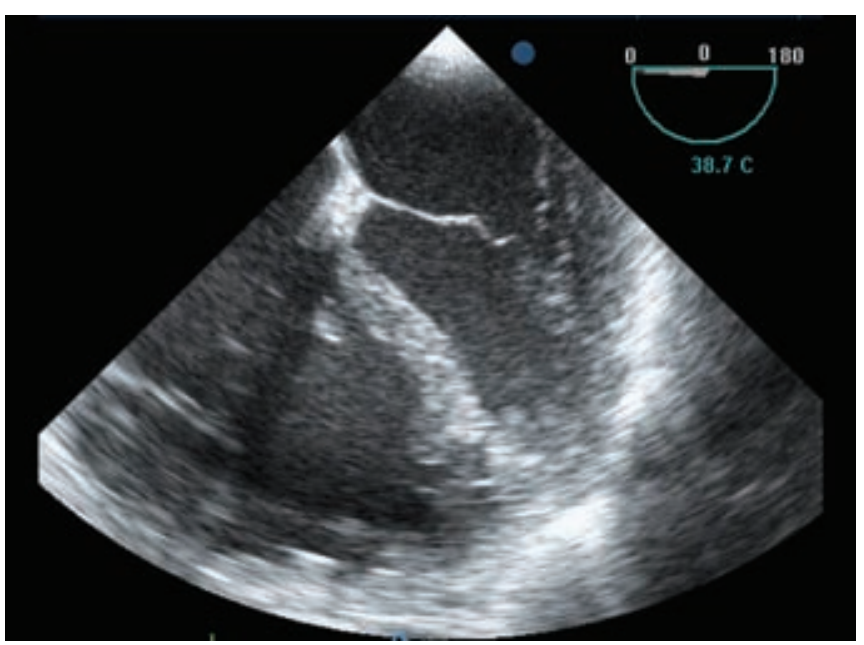

Fig. 1: Dilated RV and small LV (midesophageal four-chamber view)
Several TEE findings can provide indirect clues to deteriorating RV function. An underfilled left ventricle (LV) with distended RV is classic of RV dysfunction which can be appreciated in midesophageal and transgastric short axis views. Detailed hemodynamic calculations are usually not necessary and can be inaccurate in patients experiencing hemodynamic compromise. Increased RV/LV short axis ratio, increased RV end-diastolic and end-systolic volumes, decreased RV fractional area change and RV ejection fraction are quantitative indicators of RV dysfunction. ${ }^{4}$ Tricuspid annular motion during systole and diastole is an easily appreciated qualitative measure of RV function. ${ }^{5}$ Tricuspid annular plane systolic excursion (TAPSE) can be calculated and TAPSE less than $7.5 \mathrm{~mm}$ indicates severe RV dysfunction. Other indirect echocardiographic indicators of RV dysfunction include deviation of interventricular and interatrial septum to the left (Fig. 2), paradoxical septal wall motion, right to left shunt across the patent foramen ovale (PFO) and severe tricuspid regurgitation (TR) (see Fig. 2). ${ }^{6}$

After lung implantation and reperfusion, the decrease in RV afterload leads to improved RV function immediately and persistent RV dysfunction was associated with poor outcomes in patients with pulmonary hypertension. ${ }^{7}$

\section{PATENT FORAMEN OVALE}

PFO is present in 15 to $25 \%$ of the general population. ${ }^{8}$ Routine preoperative screening for PFO is done in LTX recipients by TTE. The PFO decompresses the RV and improves length of survival in patients with $\mathrm{PAH} .{ }^{9}$ Atrial septostomy is performed in patients with severe PAH awaiting LTX in selected centers. ${ }^{10}$ Although patient's hemodynamics improves with the existence of a PFO as mentioned above, presence of any right to left shunt can exaggerate pre-existing hypoxemia in patients with ESLD

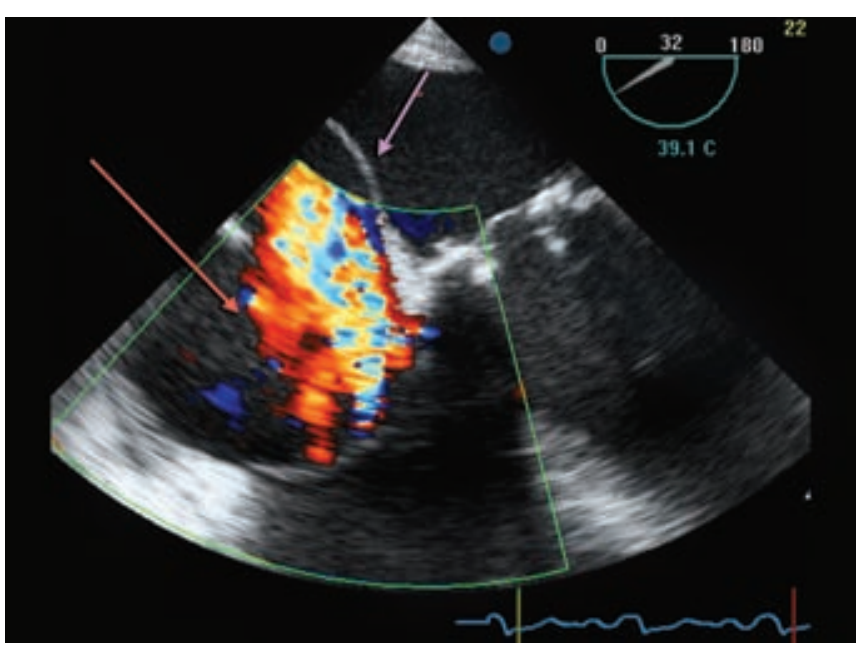

Fig. 2: Red arrow shows severe tricuspid regurgitation and pink arrow shows shift of interatrial septum to the left 
during the perioperative period. It is important to scan for a PFO by two-dimensional echocardiogram, color flow Doppler and contrast echocardiography by intraoperative TEE (Fig. 3) and communicate the findings to the surgical team. If a PFO is found during intraoperative TEE, surgical PFO closure requires $\mathrm{CPB}$ with double venous cannulation. At our institution, we have had several cases of PFO detected by intraoperative TEE but missed on preoperative TTE. There is no universal practice among our surgeons or consensus regarding the surgical closure of PFO. Large shunts are usually closed but small shunts are left alone. We also found that some pre-existing shunts could not be detected by conventional echocardiographic methods after new lung implantation in the post-bypass and postoperative period (unpublished observation). There is no study in the literature till date comparing the LTX outcomes with or without closure of PFO. Mal et al reported a case of reversal of shunt and hypoxemia with normal right atrial pressure 10 days after left lung transplantation. ${ }^{11}$ Pneumonectomy and alteration of postoperative intrathoracic anatomy were cited as reasons for delayed opening of the shunt. Device closure
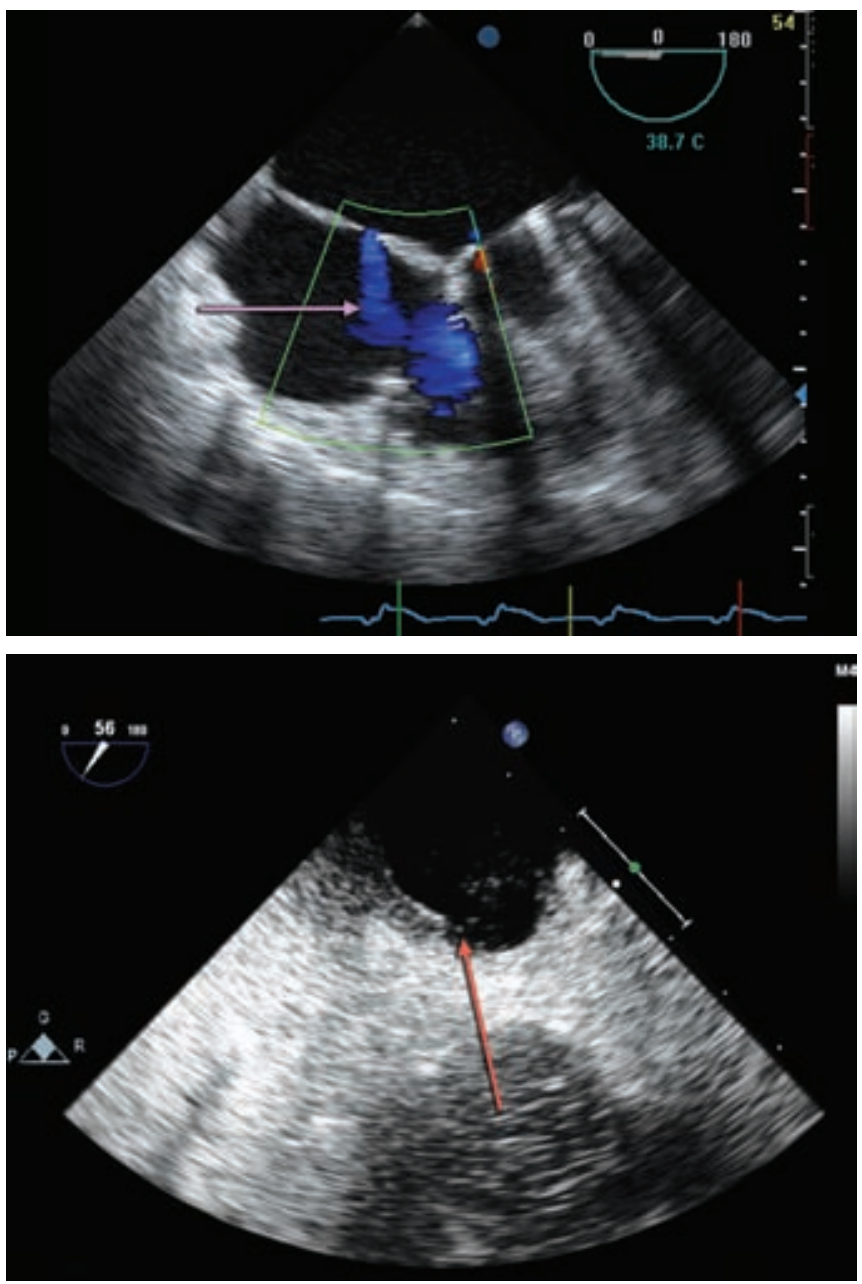

Fig. 3: Color flow Doppler and bubble study showing patent foramen ovale (arrows) can be done in the catheterization laboratory if a significant shunt is detected postoperatively.

\section{LEFT VENTRICLE}

As older patients with cardiovascular illnesses are increasingly listed for LTX, careful evaluation of LV morphology and function should be done preoperatively and by intraoperative TEE. In the study done by Puentes et al, $10 \%$ of patients had LV dysfunction and $9 \%$ had LV hypertrophy by intraoperative TEE during LTX. ${ }^{2}$ Toyooka et al evaluated left and right ventricular systolic function after living related lobar transplantation in patients with and without pulmonary hypertension. Basal LV ejection fraction was lower in PAH group compared to non-PAH group. While the RV recovered in the immediate postoperative period, LVEF recovery took 6 to 12 months to reach nonPAH group level. ${ }^{12}$

Systemic hypertension with left ventricular hypertrophy is not uncommon in elderly subjects. Associated LV diastolic dysfunction may complicate perioperative patient management. Ventricular interdependence and diastolic filling abnormalities exist in patients with $\mathrm{PAH} .{ }^{13}$ Xie et al found that LV filling takes a year to normalize after single LTX. ${ }^{14}$ Management of fluids, vasopressors and inotropes should be guided by TEE in patients with LV dysfunction. There is a lot of interest currently in the transplantation community on the effect of preoperative LV dysfunction on graft function, recovery, morbidity and mortality, which should be evaluated in future trials.

At least two studies have shown that the presence of coronary artery disease (CAD) does not affect short and medium term survival in LTX recipients. ${ }^{15,16}$ Several patients with significant $\mathrm{CAD}$ are treated with bare metal stents and undergo transplantation 6 weeks later at our institution. TEE detected regional wall motion abnormalities may indicate stent thrombosis which will necessitate an intervention in the perioperative period.

\section{TRICUSPID REGURGITATION}

LTX recipients have varying degrees of functional tricuspid regurgitation (TR) related to RV dilatation and dysfunction. Dilation of the tricuspid annulus is the mechanism for TR in patients with PAH. There is no consensus on the management of TR in patients undergoing LTX. Lung implantation results in decreased pulmonary artery pressure and thus pressure overload on RV leading to decreased TR in most patients. One study from our institution by Shigemura et al addressed the effect of combined LTX and tricuspid valve repair (TVR) in patients with severe PAH. ${ }^{17}$ They hypothesized that tricuspid annuloplasty and lung implantation result 
in decreased volume and pressure overload on the RV respectively, leading to better RV recovery. Indications for TVR included severe PAH with transpulmonary gradient $>30 \mathrm{~mm} \mathrm{Hg}$, severe TR (grade 4 on intraoperative TEE) and severe or moderate RV dysfunction. Decreased mechanical ventilation days, decreased inotropic support and lower incidence of primary graft dysfunction were demonstrated in TVR group. No difference in short term and long-term mortality could be found between the groups. However, the groups were not comparable in this retrospective study with small sample size and further controlled studies are required to provide definite answers.

\section{INCIDENTAL TEE FINDINGS}

New incidental findings have been reported with the use of intraoperative TEE. Atrial septal defect (ASD), ventricular septal defect, mitral valve lesions especially regurgitant lesions (Fig. 4), aortic valve lesions, pericardial effusion, pulmonary artery aneurysm, significant aortic atheromas (Fig. 5) and aneurysms are some of the incidental

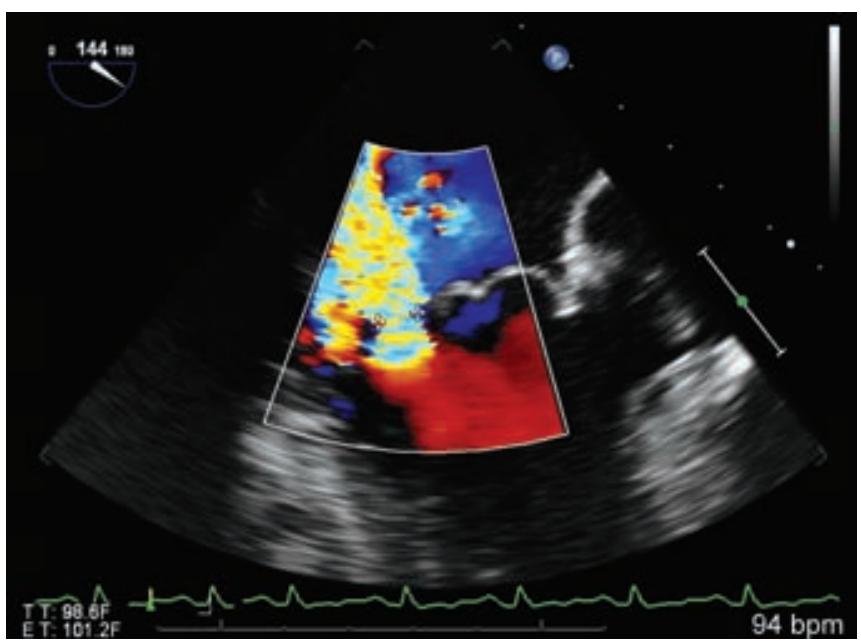

Fig. 4: Incidental finding of severe mitral regurgitation on prebypass TEE examination

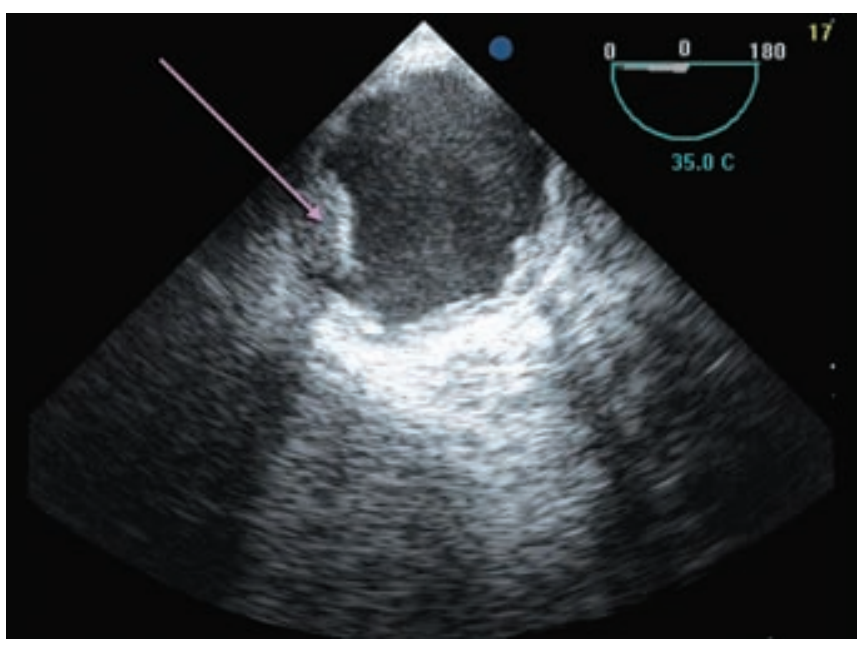

Fig. 5: Aortic atheroma in descending aorta findings. Concomitant cardiac surgery may be needed for some of these lesions along with LTX. Decision-making should be based on the severity of the lesion, its effect on cardiopulmonary function and length of bypass required to fix the additional lesions. Two studies looked at the outcome of LTX and concomitant cardiac surgery. ${ }^{18,19}$ Length of intensive care unit (ICU) stay and duration of mechanical ventilation were prolonged in one study in patients who had concomitant cardiac surgery. Long-term outcomes were not affected. At our institution, we performed aortic valve replacement, coronary bypass (both on and off pump), shunt closures and pulmonary artery aneurysm resections along with lung transplantation. Shayan et al from our institution reported double valve (mitral annuloplasty for mitral regurgitation, pulmonary commisuroplasty for pulmonary regurgitation) repair along with pulmonary artery aneurysm repair and LTX in a patient with primary PAH. ${ }^{20}$ Pulmonary artery aneurysm is diagnosed when main PA is dilated more than 29 mm (Fig. 6). Patient did well postoperatively. TEE played an important role in the diagnosis and decision making of these incidental lesions.

\section{TEE AND MECHANICAL SUPPORT}

Lung transplantation can be done off pump as sequential LTX or with the assistance of mechanical circulatory support. The choice depends on the patient factors and institutional preference. When mechanical support is needed, the type of support can be either extracorporeal membrane oxygenation (ECMO) or full CPB. ECMO support can again be either veno-venous (V-V ECMO) or veno-arterial (V-A ECMO). Sick patients with severe gas-exchange problems and supra systemic PAH are supported by ECMO preoperatively in the ICU. More often, ECMO or CPB is initiated in the operating room for the completion of the procedure. Sometimes ECMO support is required in the postoperative period because of reperfusion injury and primary graft dysfunction.

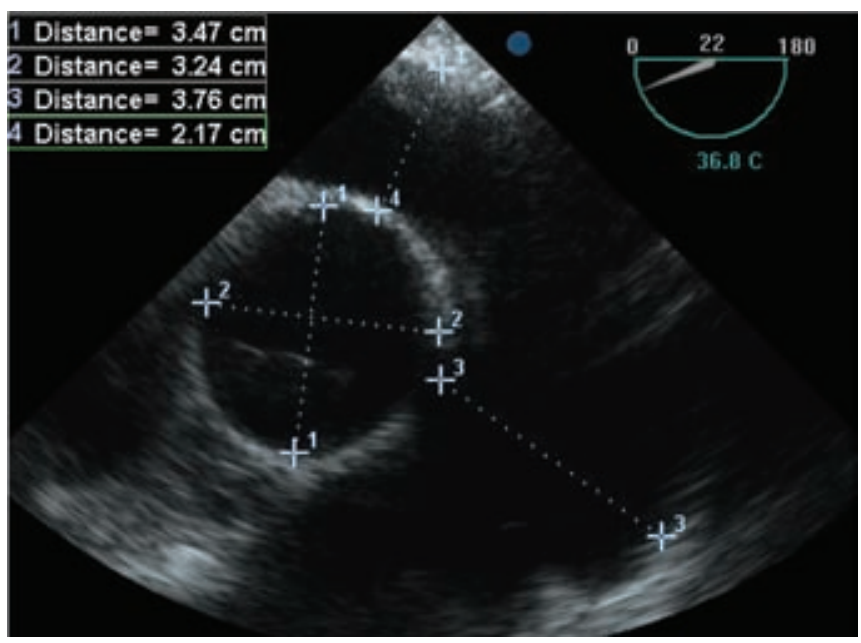

Fig. 6: Main pulmonary artery aneurysm in a patient with severe pulmonary hypertension presenting for lung transplantation $(37 \mathrm{~mm})$ 
Central or peripheral cannulation is required to initiate ECMO. In peripheral V-A ECMO, femoral artery and femoral vein are cannulated. Aorta is examined for atheromatous disease and the findings communicated to the surgeon. Guide wire is checked by TEE in the descending aorta for cannulation of femoral artery (Fig. 7). Wire and cannula are checked in the IVC for femoral venous cannulation. Venous cannula is positioned in the right atrium without impinging on interatrial septum or tricuspid valve (Fig. 8). When low flows are encountered with ECMO, TEE is useful to differentiate between hypovolemia and inadequate venous drainage because of cannula obstruction. Central cannulation (RA and aorta) is usually done in the operating room when the chest is already open for the surgical procedure. Full CPB versus ECMO support for LTX is still debated but TEE helps in cannulation for both the techniques. ${ }^{21,22}$ Veno-venous ECMO can be achieved by dual cannulation (superior vena cava by internal jugular route and inferior vena cava by femoral route) or single cannulation of the SVC with dual lumen cannula. Inflow should be located in IVC and the

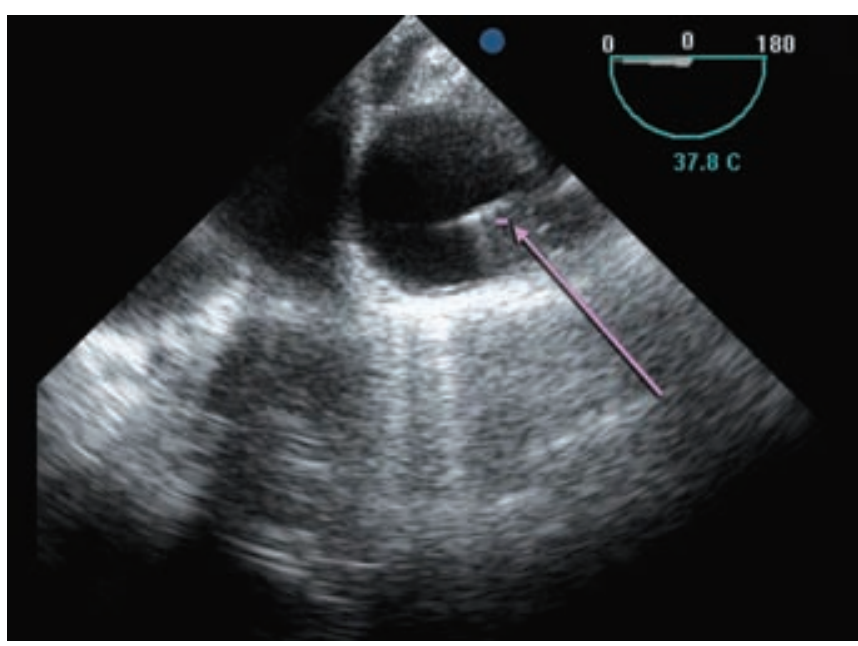

Fig. 7: Wire in the descending aorta detected by TEE to guide arterial cannulation for V-A ECMO through femoral artery

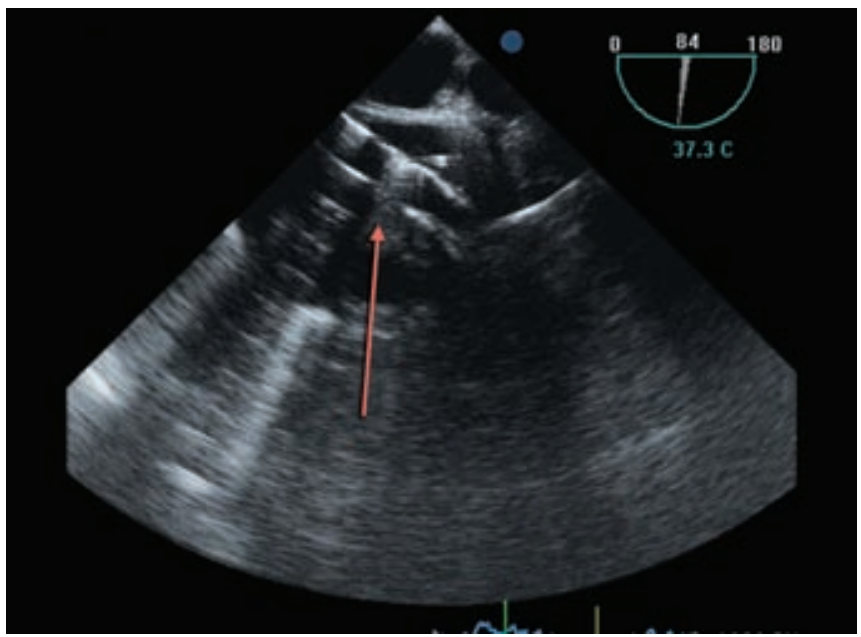

Fig. 8: Venous cannula in the right atrium (arrow) outflow in the right atrium with this dual lumen cannulation. In summary, TEE helps in cannulation and hemodynamic management of extracorporeal support for LTX recipients in the operating room and ICU.

Novalung is an interventional lung assist device which is inserted through sternotomy in the operating room. Flow is from pulmonary artery to pulmonary vein and cannulation is confirmed with TEE. Taylor et al described Novalung insertion under V-A ECMO support in a patient with suprasystemic pulmonary hypertension. ${ }^{23}$

\section{PULMONARY VASCULAR ANASTOMOSIS}

After lung implantation, TEE is used to examine pulmonary arterial and venous anastomosis and establish their flow characteristics. Right PA anastomosis can be evaluated easily by TEE but left PA is not always visualized because it's location in the blind spot of TEE. It is difficult to orient the spectral Doppler beam to the blood flow of the PA by TEE. There are no echocardiographic criteria established for the diagnosis of post lung TXP PA stenosis. In a study of 18 LTX recipients, Michel-Cherqui et al have shown that pulmonary arterial diameters at the anastomosis ranged from 1 and $1.7 \mathrm{~cm}$ by intraoperative TEE and they were considered normal. ${ }^{24}$ Stenosis should be suspected if the anastomosis diameter is $75 \%$ less than neighboring vessel along with color flow turbulence. It should be confirmed by invasive measurement of gradient across the anastomosis. Epicardial echocardiography can be used during surgery to supplement TEE in the evaluation of the pulmonary arteries especially on the left side.

Several studies addressed the intraoperative and postoperative evaluation of pulmonary veins by TEE after LTX. Some of them reported pulmonary venous thrombosis (PVT) whereas others reported venous anastomotic stenosis. Since pulmonary veins (PV) are located close to TEE probe, evaluation by two and three-dimensional echocardiography, color Doppler and spectral Doppler are possible in high percentage of patients (Figs 9A and B). Epicardial ultrasound can be used to provide additional information or verify the findings of TEE. Catena et al used epicardial echocardiography in addition to TEE to better define pulmonary venous anastomosis in nine patients. ${ }^{25}$ When TEE provided suboptimal images, they were able to obtain good images with epicardial echocardiography. Felton et al, in their study compared the visualization and measurements of pulmonary veins obtained by epicardial ultrasound and TEE. ${ }^{26}$ Left side venous visualization was better with surface ultrasound but similar measurements (diameters and velocities) were obtained with both methods.

Vessel diameter less than $0.5 \mathrm{~cm}$, velocities higher than $1 \mathrm{~m} / \mathrm{sec}$ and left atrial to pulmonary vein pressure 

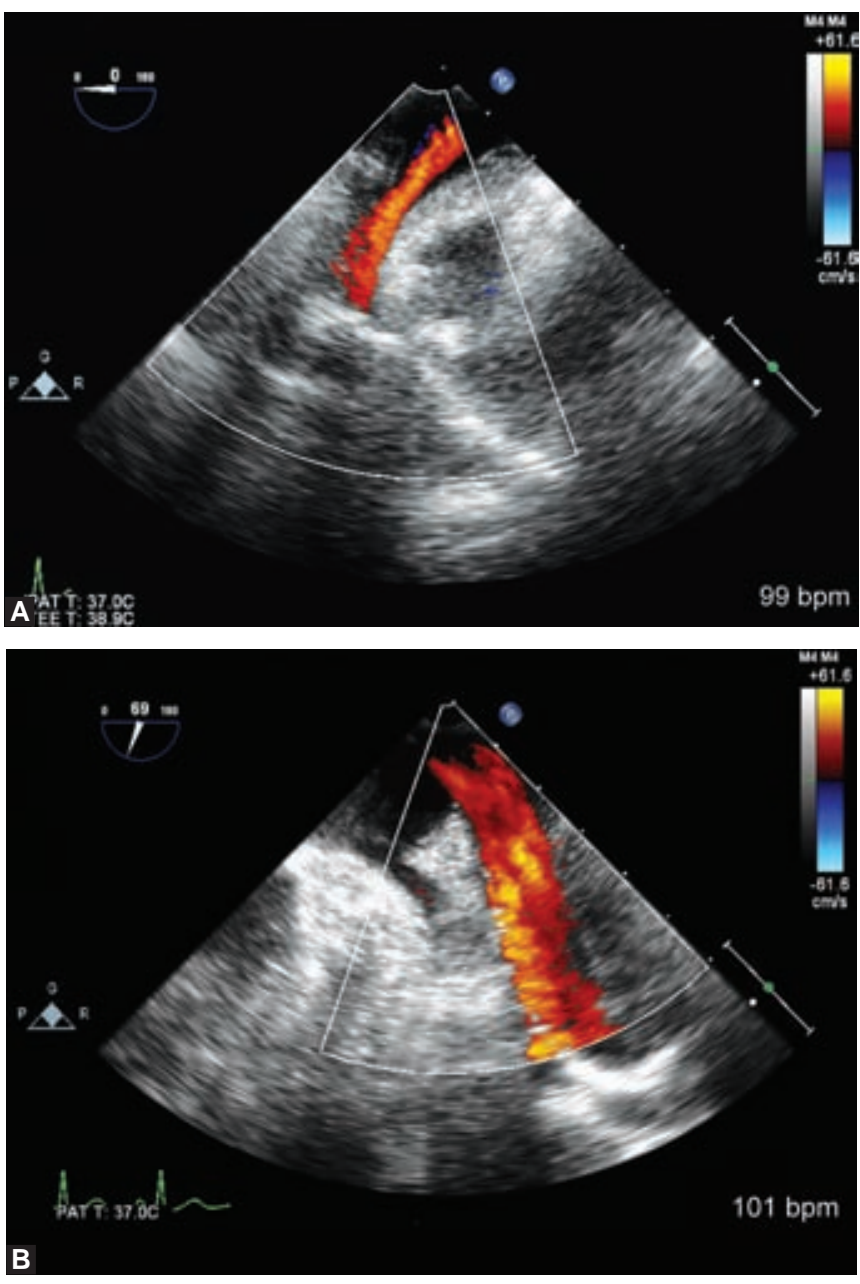

Figs 9A and B: Normal laminar flow through right $(A)$ and left (B) pulmonary veins

gradient more than 10 to $12 \mathrm{~mm} \mathrm{Hg}$ were considered as criteria for pulmonary venous stenosis. ${ }^{27}$ Vessel diameter may not always be accurate as the veins are not circular but narrowest diameter of color flow in the PV has been used to quantify stenosis. Low blood volume and proximal left atrial cuff stenosis may cause low peak systolic flow velocities (PSFV). High blood volume, contralateral arterial stenosis or donor venoconstriction can cause elevated PSFV without any PV anatomic stenosis (Fig. 10). In bilateral sequential lung implantation, PV velocities should be measured after both lungs are perfused. Clamp on the PA during second lung implantation will direct entire cardiac output to the first implanted and already reperfused lung thereby causing their increased PV systolic velocities and pressure gradients. Similarly after single LTX, since the pulmonary vascular resistance on the implanted lung is low, cardiac output may preferentially be directed toward the new lung. This may increase the PSFV in the PV of the new lung compared to the diseased lung. ${ }^{27}$ Myles reported that residual left to right shunt through atrial septal defect (ASD) increased the blood flow and PFV through the pulmonary veins. ${ }^{28}$ Increased velocity resolved after closure of ASD in their patient.

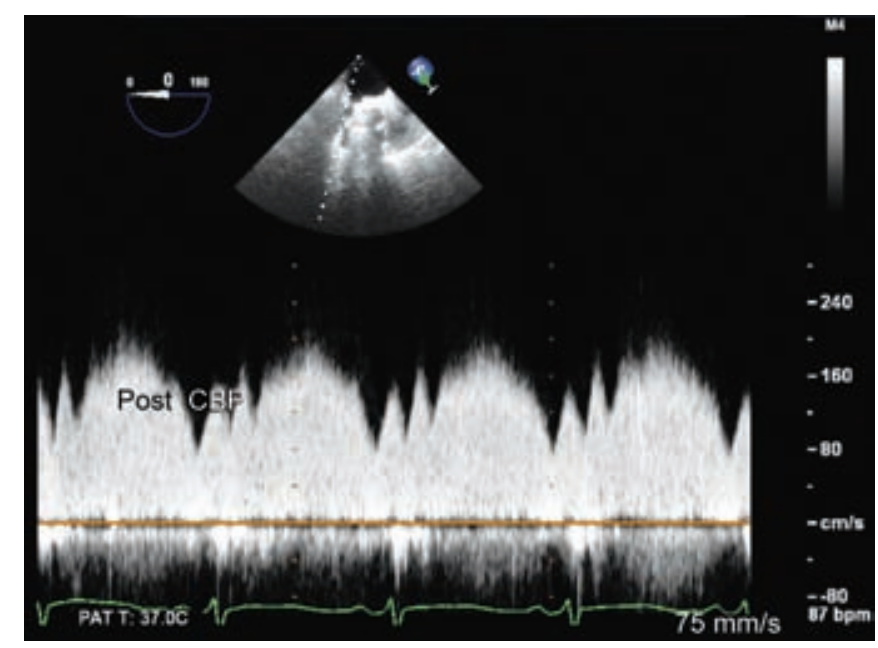

Fig. 10: High systolic flow velocity in pulmonary veins after lung transplantation should be correlated with clinical presentation

Elevated left atrial pressure from impaired ventricular (systolic or diastolic) function and mitral valve pathology (regurgitation) can also affect PSFV measurement in the pulmonary veins.

Miyaji et al studied flow velocity index (ratio of flow velocities on both sides) in 17 patients undergoing living related lobar transplantation and they predicted success of transplantation by the equivalence of flow velocities in both PV. Differential flow velocity may indicate pulmonary vascular stenosis. ${ }^{29}$ Michel-Cherqui et al in their study of 18 lung LTX recipients, TEE revealed higher PSFV in the pulmonary venous system of five patients. ${ }^{24}$ Only patient required reoperation for early graft failure. TEE findings should be correlated with clinical course in the postoperative period. Huang et al reported graft failure secondary to stenosis at the pulmonary venous anastomosis (0.25 cm diameter). Mosaic pattern on color flow Doppler and higher gradient $(>1.6 \mathrm{~m} / \mathrm{sec})$ on pulse wave Doppler were demonstrated in their patient. ${ }^{30}$ Surgical repair could not be done because of inadequate donor tissue available for anastomosis. This case described the importance of TEE examination post-lung implantation. Pazos-Lopez et al reported a case of pulmonary vein stenosis diagnosed 11 days after lung transplantation by TEE. Stenosis was successfully treated with stent implantation and peak gradient across the stenosis $\left(25 \mathrm{~mm} \mathrm{Hg}\right.$ ) dropped to $5 \mathrm{~mm} \mathrm{Hg}$ after stenting. ${ }^{31}$ Since clinical manifestations of pulmonary venous stenosis are similar to primary graft dysfunction and reperfusion injury, TEE examination in the operating room and ICU is mandatory and provides definite diagnosis.

Pulmonary venous thrombosis (PVT) is another pathology, which can present with similar clinical features to pulmonary venous stenosis. Intraoperative TEE can provide rapid diagnosis and leads to immediate surgical intervention. Cywinski et al reported a case of PVT after 
sequential double lung transplantation. This was diagnosed by intraoperative TEE and treated successfully by surgical thrombectomy. ${ }^{32}$ Mcllroy et al reported three cases of PVT diagnosed by intraoperative TEE in their series of 81 lung transplantations (3.6\%). All patients underwent immediate surgical thrombectomy. ${ }^{33}$ Schulman et al reported a $15 \%$ incidence of PVT in their series of lung transplantation and their 90-day mortality in patients with PVT was 38\%. TEE was performed within 48 hours of LTX to diagnose PV obstruction. ${ }^{34}$ Incidence of PVT after LTX varied (3-25\%) in different reports. Diagnosis of PVT by intraoperative TEE provides the surgeon an opportunity to go back on CPB and extract the thrombus immediately. Delay in diagnosis to the postoperative period creates a dilemma whether to treat the thrombosis by conservative management, anticoagulation, thrombolysis or by immediate re-exploration. Larger thrombus $(>1.6 \mathrm{~cm})$ and greater acceleration of PSFV are associated with poor outcome and will require surgical intervention. In contrast, small thrombus with no flow acceleration can be managed conservatively. Lobectomy/ pneumonectomy and retransplantation may be needed in severe lung injury. PVT after single lung transplant is obviously more serious because of lack of compensation from the other diseased lung. Although CT scanning, MRI scanning and angiography can establish postoperative diagnosis of PVT, TEE is an easily available bedside tool and provides early diagnosis in unstable patients, who cannot be transported to the imaging facilities.

\section{OTHER INTRAOPERATIVE USES}

TEE provides guidance in the placement of Swan-Ganz catheters in PA as blind insertion is often difficult and can cause arrhythmias in patients with dilated RV. It is important to make sure PA catheter is positioned in the main PA as it can be clamped and cut during pneumonectomy if it is inserted distally into right or left PA (Figs 11A and B). If there is a failure or difficulty in floating PA catheter in spite of repeated attempts, it is possible to measure RV systolic pressure from TR jet by continuous wave Doppler (Fig. 12).

Several surgical manipulations affect hemodynamics and systemic perfusion pressure especially during off-pump LTX. Dissection of the lung, PA clamping, surgical bleeding, hypovolemia, retraction of the heart during anastomosis, systemic inflammatory response, metabolic and respiratory acidosis, PA unclamping and lung reperfusion, coagulopathy and chest closure are few reasons for the fall in systemic blood pressure. Cardiac filling pressures cannot be relied upon as they are altered by position of the patient, retraction of the heart and PA clamping. Direct visualization of the heart by TEE provides useful information as to the etiology of hypotension and directs appropriate management.
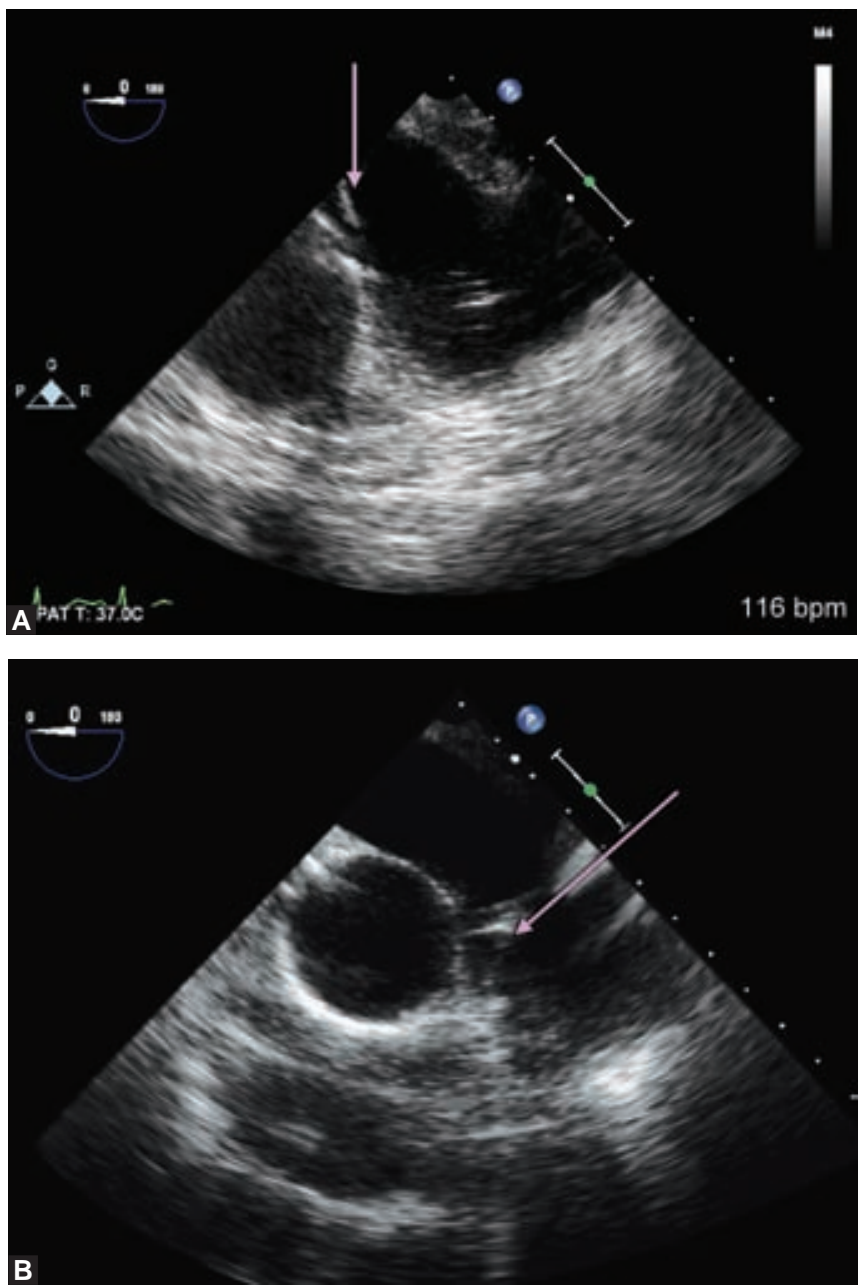

Figs 11A and B: Swan-Ganz catheter in right PA

(A) pulled back to $(B)$ before PA clamping

Air embolism is a fatal and infrequently reported complication during lung transplantation. ${ }^{35,36}$ Inadequate de-airing of graft vasculature, accidental ventricular tear and central vein insertion are few reported reasons for air embolism during lung transplantation. TEE is the most sensitive monitor for detection of air-embolism and provides early diagnosis.

\section{POSTOPERATIVE TEE}

Hemodynamic instability after lung transplantation could be related to bleeding causing hemomediastinum or hemothorax (Fig. 13). TEE establishes immediate diagnosis. TEE also helps in the diagnosis of cardiac causes (acute myocardial infarction, pulmonary embolism (Fig. 14) and right ventricular failure) of hemodynamic instability. As described in the previous sections, two causes of impaired oxygenation after lung transplantation namely, right to left shunt and pulmonary venous obstruction can be diagnosed at the bedside using TEE. TEE is extremely useful in weaning patients from mechanical circulatory support, such as V-A ECMO, postoperatively at the bedside. Patients are taken back to the operating room for decannulation. TEE findings 

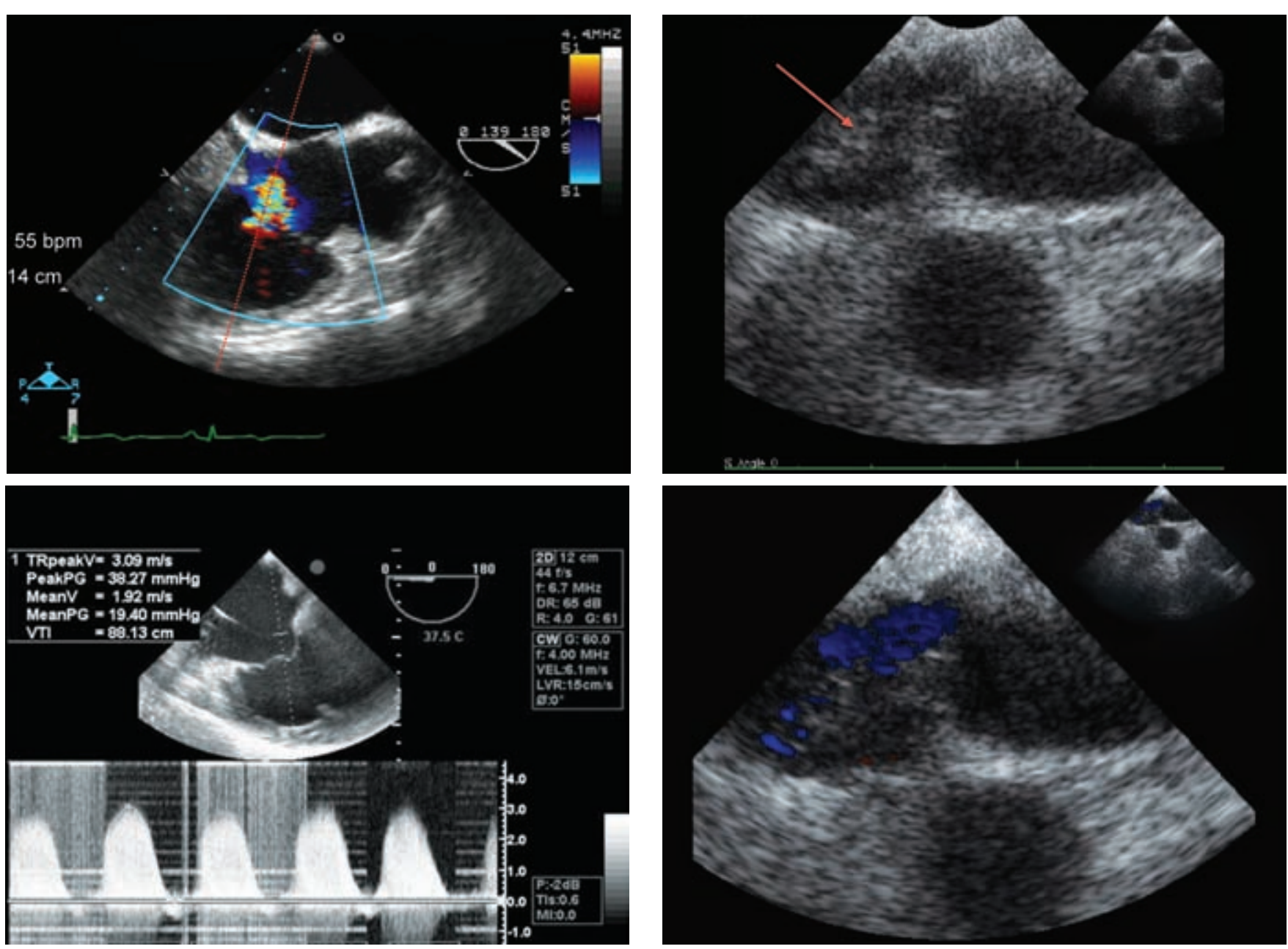

Fig. 12: Continuous wave Doppler through tricuspid regurgitant jet to measure RV systolic pressure
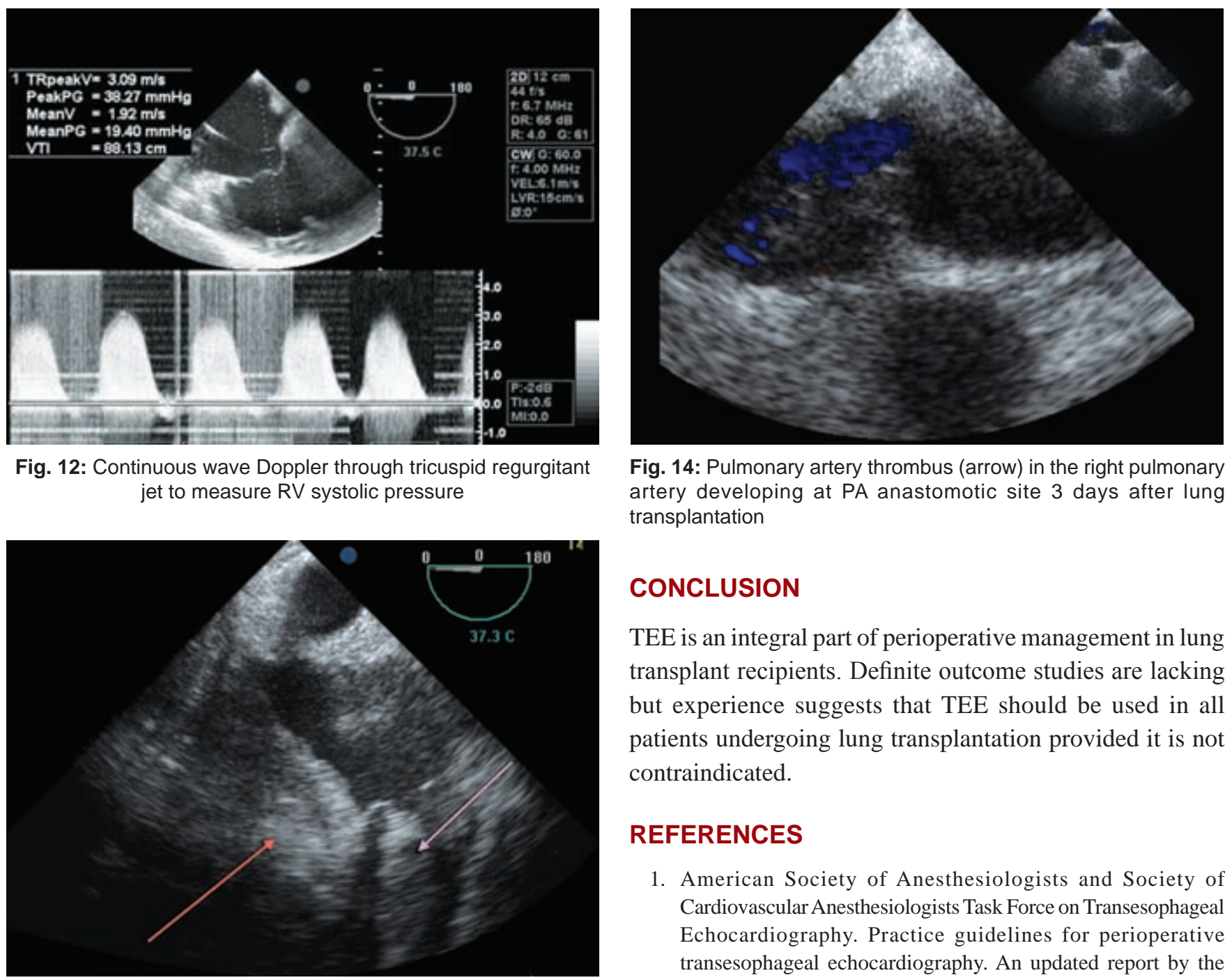

Fig. 14: Pulmonary artery thrombus (arrow) in the right pulmonary artery developing at PA anastomotic site 3 days after lung transplantation

\section{CONCLUSION}

TEE is an integral part of perioperative management in lung transplant recipients. Definite outcome studies are lacking but experience suggests that TEE should be used in all patients undergoing lung transplantation provided it is not contraindicated.

\section{REFERENCES}

1. American Society of Anesthesiologists and Society of Cardiovascular Anesthesiologists Task Force on Transesophageal Echocardiography. Practice guidelines for perioperative transesophageal echocardiography. An updated report by the American Society of Anesthesiologists and the Society of Cardiovascular Anesthesiologists Task Force on Transesophageal Echocardiography. Anesthesiology 2010;112:1084-1096.

Fig. 13: Hemothorax complicating lung transplantation: Red arrow indicates lung and pink arrow indicates hemothorax

(good biventricular function and adequate ventricular filling) are correlated with hemodynamics, mixed venous oxygen saturation and arterial blood gas before decannulation. Sometimes V-A ECMO is converted to V-V ECMO provided the cardiac function is normal with the patients requiring only support for oxygenation.

2. Puentes W, Schwabl P, Slinger P, KeshavjeeS, Meineri M. Impact of transesophageal echocardiography on surgical decision making during lung transplant. SCA Thoracic Anesthesia Symposium Abstract 2013 (Unpublished).

3. Gorcsan J 3rd, Edwards TD, Ziady GM, Katz WE, Griffith BP. Transesophageal echocardiography to evaluate patients with severe pulmonary hypertension for lung transplantation. Ann Thorac Surg 1995;59:717-722. 
4. Meineri M, Van Rensburg AE, Vegas A. Right ventricular failure after LVAD implantation: prevention and treatment. Best Pract Res Clin Anaesthesiol 2012;26:217-229.

5. Sato T, Tsujino I, Ohira H, et al. Validation study on the accuracy of echocardiographic measurements of right ventricular systolic function in pulmonary hypertension. J Am Soc Echocardiogr 2012;25:280-286.

6. Catena E, Tasca G. Role of echocardiography in the perioperative management of mechanical circulatory assistance. Best Pract Res Clin Anaesthesiol 2012;26:199-216.

7. Katz WE, Gasior TA, Quinlan JJ, Lazar JM, Firestone L, Griffith BP, Gorcsan J 3rd. Immediate effects of lung transplantation on right ventricular morphology and function in patients with variable degrees of pulmonary hypertension. J Am Coll Cardiol 1996;27:384-391.

8. Di Tullio MR, Jin Z, Russo C, et al. Patent foramen ovale, subclinical cerebrovascular disease, and ischemic stroke in a population-based cohort. J Am Coll Cardiol 2013;62:35-41.

9. Glanville AR, Burke C, Theodore J, et al. Primary pulmonary hypertension: length of survival in patients referred for heartlung transplantation. Chest 1987;91:675-681.

10. Judge EP, Gaine SP. Management of pulmonary arterial hypertension. Curr Opin Crit Care 2013;19:44-50.

11. Mal H, Biondi G, Gaudry S, et al. Delayed reopening of a hemodynamically significant patent foramen ovale after left lung transplantation: emergency management. J Heart Lung Transplant 2010;29:224-227.

12. Toyooka S, Kusano KF, Goto K, et al. Right but not left ventricular function recovers early after living-donor lobar lung transplantation in patients with pulmonary arterial hypertension.J Thorac Cardiovasc Surg 2009;138:222-226.

13. Stojnic BB, Brecker SJ, Xiao HB, et al. Left ventricular filling characteristics in pulmonary hypertension: A new mode of ventricular interaction. Br Heart J 1992;68:16-20.

14. Xie GY, Lin CS, Preston HM, et al. Assessment of left ventricular diastolic function after single lung transplantation in patients with severe pulmonary hypertension.Chest 1998;114:477-481.

15. Sherman W, Rabkin DG, Ross D, et al. Lung transplantation and coronary artery disease. Ann Thorac Surg 2011;92:303-308.

16. Choong CK, Meyers BF, Guthrie TJ, et al. Does the presence of preoperative mild or moderate coronary artery disease affect the outcomes of lung transplantation? Ann Thorac Surg 2006; 82:1038-1042.

17. Parekh K, Meyers BF, Patterson GA, et al. Outcome of lung transplantation for patients requiring concomitant cardiac surgery. J Thorac Cardiovasc Surg 2005;130:859-863.

18. Johnson SB, Allred AM, Cline AM, et al. Cardiac procedures in lung transplant recipients do not increase mortality in selected patients. Ann Thorac Surg 2006;82:460-463.

19. Shayan H, Sareyyupoglu B, Shigemura N, et al. Lung transplant, double valve repair, and pulmonary artery aneurysm resection. Ann Thorac Surg 2012;93:e3-5.

20. Shigemura N, Sareyyupoglu B, Bhama J, et al. Combining tricuspid valve repair with double lung transplantation in patients with severe pulmonary hypertension, tricuspid regurgitation, and right ventricular dysfunction. Chest 2011;140:1033-1039.

21. Ius F, Kuehn C, Tudorache I, et al. Lung transplantation on cardiopulmonary support: venoarterial extracorporeal membrane oxygenation outperformed cardiopulmonary bypass. J Thorac Cardiovasc Surg 2012;144:1510-1516.

22. Bittner HB, Binner C, Lehmann S, et al. Replacing cardiopulmonary bypass with extracorporeal membrane oxygenation in lung transplantation operations. Eur J Cardiothorac Surg 2007;31: 462-467.
23. Taylor K, Holtby H. Emergency interventional lung assist for pulmonary hypertension. Anesth Analg 2009;109:382-385.

24. Michel-Cherqui M, Brusset A, Liu N, et al. Intraoperative transesophageal echocardiographic assessment of vascular anastomoses in lung transplantation. A report on 18 cases. Chest 1997;111:1229-1235.

25. Catena E, Paino R, Fieschi S, et al. Lung transplantation and pulmonary vein thrombosis: a possible role of epicardial echocardiography. J Cardiothorac Vasc Anesth 2008;22:167-168.

26. Felten ML, Michel-Cherqui M, Sage E, Fischler M. Transesophageal and contact ultrasound echographic assessments of pulmonary vessels in bilateral lung transplantation. Ann Thorac Surg 2012;93:1094-1100.

27. González-Fernández C, González-Castro A, RodríguezBorregán JC, et al. Pulmonary venous obstruction after lung transplantation. Diagnostic advantages of transesophageal echocardiography. Clin Transplant 2009;23:975-980.

28. Myles PS, Marasco S. Misleading turbulent flow through pulmonary venous anastomoses during lung transplantation. Anesth Analg 2008;107:1504-1505.

29. Miyaji K, Matsubara H, Nakamura K, et al. Equivalence of flow velocities through bilateral pulmonary vein anastomoses in bilateral living-donor lobar lung transplantation. J Heart Lung Transplant 2005;24:860-864.

30. Huang YC, Cheng YJ, Lin YH, Wang MJ, Tsai SK. Graft failure caused by pulmonary venous obstruction diagnosed by intraoperative transesophageal echocardiography during lung transplantation. Anesth Analg 2000;91:558-560.

31. Pazos-López P, Piñeiro-Portela M, Bouzas-Mosquera A, et al. Images in cardiovascular disease. Pulmonary vein stenosis after lung transplantation successfully treated with stent implantation. Circulation 2010 21;122:2745-2747.

32. Cywinski JB, Wallace L, Parker BM. Pulmonary vein thrombosis after sequential double-lung transplantation. J Cardiothorac Vasc Anesth 2005;19:225-227.

33. Schulman LL, Anandarangam T, Leibowitz DW, et al. Four-year prospective study of pulmonary venous thrombosis after lung transplantation. J Am Soc Echocardiogr 2001;14:806-812.

34. McIlroy DR, Sesto AC, Buckland MR. Pulmonary vein thrombosis, lung transplantation, and intraoperative transesophageal echocardiography. J Cardiothorac Vasc Anesth 2006;20:712-715.

35. Le Guen M, Trebbia G, Sage E, Cerf C, Fischler M. Intraoperative cerebral air embolism during lung transplantation: treatment with early hyperbaric oxygen therapy. J Cardiothorac Vasc Anesth 2012 Dec;26(6):1077-1079.

36. Higuchi T, Oto T, Millar IL, et al. Preliminary report of the safety and efficacy of hyperbaric oxygen therapy for specific complications of lung transplantation. J Heart Lung Transplant 2006;25:1302-1309.

\section{ABOUT THE AUTHORS}

\section{Kathirvel Subramaniam (Corresponding Author)}

Clinical Associate Professor, Department of Anesthesiology, UPMC Presbyterian Hospital, Pittsburgh, PA, USA, Phone: 0014126475635 e-mail: subramaniamk@upmc.edu

\section{Stephen A Esper}

Assistant Professor, Department of Anesthesiology, UPMC Presbyterian Hospital, Pittsburgh, PA, USA 